1 Universidade Federal do Oeste do Pará (Ufopa), Instituto de Saúde Coletiva (Isco) - Santarém (PA), Brasil.

laralissa_@hotmail.com

2 Universidade do Estado do Pará (Uepa) - Santarém (PA), Brasil.

\section{Acesso e acolhimento na Atenção Básica da região Oeste do Pará}

\author{
Access and embracement in the Primary Care of the Western region \\ of Pará
}

Larissa Ádna Neves Silva1, Rui Massato Harayama', Franciane de Paula Fernandes², Juliana Gagno Lima1

DOI: $10.1590 / 0103-1104201912207$

RESUMO O artigo teve como objetivo geral analisar as condições de acesso e acolhimento da Atenção Básica na região de saúde do Baixo Amazonas, localizada no Oeste do Pará, sob a perspectiva das equipes de saúde e dos usuários. Trata-se de uma pesquisa descritiva e quantitativa, na qual foram utilizados dados secundários do segundo ciclo do PMAQ-AB, ano 2014. Foram selecionadas variáveis do Instrumento de Avaliação Externa dos módulos II (profissional) e III (usuários). Consideraram-se 58 equipes de saúde e 232 usuários de 11 municípios da região de saúde. Alguns avanços notáveis no processo de trabalho das equipes estão relacionados com formas de agendamento, Unidades Básicas de Saúde (UBS) próximas aos domicílios dos usuários, horário de funcionamento e implantação do acolhimento. Contudo, há dificuldades na organização da agenda e necessidade de fichas e fila para que o usuário chegue ao atendimento. A pesquisa revelou alta porcentagem de implantação do acolhimento como parte do cotidiano de trabalho das equipes, entretanto, cabe avaliar não apenas a existência, mas também a qualidade do acolhimento, visto que este é mais do que uma triagem ao médico. De modo geral, ainda persistem obstáculos no processo de escuta e na resolutividade das necessidades de saúde dos usuários.

PALAVRAS-CHAVE Atenção Primária à Saúde. Acesso aos serviços de saúde. Acolhimento.

\begin{abstract}
The article aims to analyze the conditions of access and embracement of Primary Care in the health region called Baixo Amazonas, located in Western Pará, from the perspective of health teams and patients. This is a descriptive and quantitative research, which used secondary data from the second cycle of PMAQ-AB, in the year 2014. Variables from the External Evaluation Instrument of modules II (professional) and III (patients) were selected. 58 health teams and 232 users from 11 municipalities in the health region were considered. Some notable advances in the work process of teams are related to scheduling, UBS close to patients homes, opening hours and implementation of the embracement. However, there are difficulties in organizing the agenda as well as the need of tokens and queue for the user to reach the service. The research revealed a high percentage of implantation of the embracement as part of the teams' daily work, however, it is important to evaluate not only the existence, but the quality of the embracement, since it is more than a screening to the doctor. In general, obstacles still persist in the listening process and in resolving the health needs of patients.
\end{abstract}

KEYWORDS Primary Health Care. Access to health services. Embracement. 


\section{Introdução}

A discussão de acesso e acolhimento em contexto regional insere-se em uma nova perspectiva no modelo assistencial, pautada na integração dos serviços do Sistema Único de Saúde (SUS) e respostas compatíveis às necessidades de saúde da população. Considerando a diversidade de abordagens dos conceitos de acesso e acolhimento, destacam-se as definições de Starfield $\mathbf{1}$ e Franco et al. ${ }^{2}$. Starfield $\mathbf{1}$ aborda a discussão de acesso a partir do uso oportuno dos serviços para o alcance dos melhores resultados possíveis, em termos de tempo e atendimento às necessidades de saúde. Envolve a experiência de uso do cidadão ao buscar o serviço de saúde como sua referência e associa cuidado com necessidades, ampliando o olhar do aspecto geográfico. Sobre o acolhimento, Franco et al. ${ }^{2}$ apontam este dispositivo como uma possibilidade de aperfeiçoamento (ou inversão) da lógica de organização dos serviços de saúde, com foco na relação equipe de saúde-usuário. Para os autores, três aspectos são definidores para a compreensão do acolhimento: a universalidade, a presença de equipe multiprofissional e a base da humanização e cidadania nas relações de cuidado, nos encontros da equipe de saúde e a comunidade e nos processos de escuta.

A garantia do acesso e do acolhimento é de suma importância para a consolidação do direito universal da população ao SUS e demanda uma estratégia de mudança no processo de trabalho, no qual procura estabelecer uma relação de vínculo e confiança entre profissionais/serviço/usuários, no intuito de, coletivamente, chegarem a soluções conjuntas para resolução das necessidades de saúde e, principalmente, ao alcance do princípio do SUS denominado integralidade ${ }^{3}$.

Nos últimos anos, o debate sobre região de saúde tem-se expandido, a despeito de unir o que o modelo de descentralização brasileiro fragmentou, bem como garantir o que o arcabouço constitucional do SUS diz sobre a construção de serviços em rede regionalizada e hierarquizada. Autores colocam que o fato de as leis não terem sido suficientes para proteger o sistema do clientelismo privatista e ineficiente fez com que o foco fosse direcionado para o município como o núcleo organizacional do sistema. Contudo, constituir a execução municipal trouxe, de forma associada, uma dificuldade da integração em rede de políticas, programas e serviços entre os entes federativos, gerando iniquidades no sistema e em suas redes locais. Garantir a integralidade não é algo de fácil obtenção, principalmente em um município isolado ${ }^{4,5}$.

A regionalização em saúde é vista como o meio para superar as desigualdades no acesso e para adequar os meios de oferta de serviços, de modo que ocorra compartilhamento solidário das responsabilidades entre os atores envolvidos na organização da região e redes, constituindo assim uma visão mais abrangente da realidade do que a meramente administrativa ${ }^{6,7}$. No Brasil, o processo de regionalização é fenômeno complexo, devido ao modelo federativo que apresenta em seu arsenal uma série de desigualdades socioespaciais; e focar exclusivamente nos municípios para garantia do acesso à saúde é insuficiente considerando a complexidade de uma rede de atenção à saúde muitas vezes fragmentada e inexistente ${ }^{4}$. Um dos exemplos da importância de 'regionalizar a descentralização' é o processo de referenciamento dos usuários para outros serviços. Com o estabelecimento interfederativo, o ente de maior porte populacional e econômico deve responder pelos serviços de maior complexidade, sendo esse referência tanto para os outros municípios quanto para a população do município (dentro da região) ${ }^{8}$.

Pesquisas demonstram que a região Norte apresenta um baixo desenvolvimento econômico e baixa oferta de serviços, denotando a fragilidade na universalidade do acesso. Em um estudo realizado na região de saúde Entorno de Manaus, dados sobre as distâncias e meios de acesso entre os municípios apontam que o deslocamento preferencial é feito por via fluvial e aérea, demonstrando que o modelo 
de interação da região não é rede. Além dessa característica que é um limitador de acesso, destaca a desproporção geográfica existente entre o município-sede e os demais municípios da regional, que são marcados por situações de pobreza e indicadores sociais insatisfatórios ${ }^{6,7}$. Tais informações também caracterizam a região de saúde do Baixo Amazonas, que se apresenta em um contexto sociodemográfico diferenciado e que merece atenção em suas especificidades.

Em outras palavras, encontram-se muitos desafios para a efetivação das regiões de saúde, entre eles: dificuldades de identificar mecanismos de articulação, principalmente pelo Estado, necessidades de acordos solidários para municípios com baixa oferta de serviços e baixos índices socioeconômicos e fragilidades na construção dos Colegiados de Gestão Regional ${ }^{9}$. Dessa forma, vê-se a importância de pensar em políticas adequadas aos contextos regionais, que a abordagem em saúde seja concebida de forma intersetorial, que ocorra intensa articulação interfederativa (União, estados e municípios) e o reconhecimento do papel do Estado na rede de atenção, para que as responsabilidades e distribuição dos serviços sejam, de fato, ofertados de forma equânime no SUS.

Assim, este trabalho buscou analisar as condições de acesso e acolhimento da Atenção Básica ( $\mathrm{AB}$ ) na região de saúde do Baixo Amazonas, Pará, sob a perspectiva das equipes de saúde e dos usuários.

\section{Material e métodos}

O estudo trata-se de uma pesquisa do tipo descritiva, com abordagem quantitativa, no qual foram utilizadas as bases de dados secundários do Programa Nacional de Melhoria do Acesso e da Qualidade da Atenção Básica (PMAQ-AB), de acesso público, da região de saúde do Baixo Amazonas, localizada no Oeste do Pará.

O locus de análise dos dados do trabalho foi a região de saúde do Baixo Amazonas, uma das 13 regiões de saúde do estado do Pará, que possui uma população total de cerca de 733.437 habitantes, composta por 14 municípios: Oriximiná, Óbidos, Alenquer, Almeirim, Monte Alegre, Prainha, Santarém, Juruti, Terra Santa, Faro, Mojuí dos Campos, Placas, Curuá e Belterra, sendo a maior parte desses municípios com população inferior a $50 \mathrm{mil}$ habitantes ${ }^{\mathbf{1 0}}$. Dos municípios da região, Faro, Mojuí dos Campos e Oriximiná não aderiram ao segundo ciclo do PMAQ-AB no ano de 2014.

O PMAQ-AB tem como objetivo induzir os processos avaliativos na $\mathrm{AB}$, de modo que procura estimular e valorizar o profissional da saúde que atua em equipes multiprofissionais no âmbito da $A B$ e da Estratégia Saúde da Família, elevando o repasse de recursos do incentivo federal para os municípios participantes que atingirem melhora na qualidade do atendimento, bem como ampliar o acesso e garantir um padrão de qualidade comparável nacional, regional e localmente, de maneira a permitir maior transparência e efetividade das ações governamentais direcionadas à $\mathrm{AB}^{11}$.

O programa está organizado em quatro fases complementares: 1) Adesão e Contratualização; 2) Desenvolvimento; 3) Avaliação Externa; e 4) Recontratualização. Na avaliação externa, $o$ instrumento de coleta apresenta seis módulos: 1) Módulo I (observação da Unidade Básica de Saúde - UBS), 2) Módulo II (entrevista com o profissional), 3) Módulo III (entrevista com usuários) 4) Módulo IV (entrevista com o Núcleo de Apoio e Saúde da Família - Nasf); e 5) Módulos V e VI (a infraestrutura e processo de trabalho das equipes de saúde bucal) ${ }^{\mathbf{1 2}}$.

Nesta pesquisa, foram utilizados exclusivamente os dados secundários do segundo ciclo do PMAQ-AB (2014) referentes às equipes de saúde da família, por meio de uma seleção de variáveis dos módulos II (entrevista com o profissional) e III (entrevista com usuários) do Instrumento de Avaliação Externa do PMAQ11. Os dados foram obtidos por meio do site do Ministério da Saúde, seção PMAQ-AB do Departamento de Atenção Básica, que tem acesso público: microdados de acesso público do ciclo 2 do PMAQ-AB, 
com dados sobre o estado do Pará, disponível em: http://dab.saude.gov.br/portaldab/ ape_pmaq.php?conteudo=2_ciclo. Esses dados foram disponibilizados em planilha Excel $^{\circledR}$ pelo sistema, que foram importados para o programa Statistical Package for Social Science for Windows ${ }^{\circledR}$ (SPSS 22.0).

No SPSS 22.0, os dados de cada município foram agregados para visualização da região de saúde, sendo filtrada dos dados do estado do Pará. As variáveis selecionadas foram rotuladas, ou seja, foram acrescentados os rótulos de perguntas e respostas do Instrumento de Avaliação Externa do PMAQ-AB no programa SPSS, e as tabelas foram geradas. Foram consideradas 58 equipes de saúde dos 11 municípios do Baixo Amazonas (PA) que aderiram ao PMAQ-AB em 2014, cada qual com 1 profissional de nível superior respondente; e 232 usuários participantes do PMAQ-AB, que foram entrevistados sobre a experiência de uso das UBS.

As tabulações dos resultados envolveram frequência simples e percentual com tabelas organizadas a partir das variáveis selecionadas sobre acesso e acolhimento, resposta dos profissionais e dos usuários. Foram desconsideradas as alternativas com resposta 'não se aplica', sendo recalculadas as porcentagens geradas pelo SPSS 22.0. Algumas observações foram acrescidas ao final das tabelas, tais como, questões com resposta 'não sabe/não respondeu' e questões que admitem múltiplas respostas.

Por se tratar de uma pesquisa com base em dados quantitativos de banco de dados de acesso público, não houve necessidade de submissão do projeto de pesquisa ao Comitê de Ética e Pesquisa, de acordo com a Resolução $\mathrm{n}^{\circ} 510$, de 07 de abril de 2016.

\section{Resultados e discussão}

A tabela 1 apresenta dados gerais e do PMAQ-AB referentes à população, equipes de saúde por município, equipes participantes do PMAQ-AB e usuários entrevistados pelo programa. Os resultados mostram que os municípios da região de saúde do Baixo Amazonas apresentam população entre $10 \mathrm{e}$ 60 mil habitantes, menos Santarém, que tem mais de 290 mil habitantes, conferindo-se como o município-polo da região. Entre as equipes, observa-se que, mesmo no cenário de possibilidades de adesão ao programa, alguns municípios, como Curuá, Monte Alegre e Óbidos, apresentam-se com menos de 50\% de equipes participantes até o segundo ciclo do PMAQ-AB. Além disso, os municípios de Faro, Mojuí dos Campos e Oriximiná não aderiram ao PMAQ-AB. Quanto aos usuários, a quantidade prevista de quatro usuários por equipe encontra-se exata/sem perda.

Entre as equipes de saúde participantes do PMAQ-AB da região do Baixo Amazonas, observa-se o quantitativo de 58 equipes, distribuídas nos municípios conforme tabela 1. Por meio de variáveis específicas, foram analisados no eixo acesso: formas de agendamento, tempos de espera ao chegar e para a consulta, e atendimento à demanda espontânea. 
Tabela 1. Distribuição geral de equipes de saúde e usuários na região de saúde do Baixo Amazonas, Pará

\begin{tabular}{|c|c|c|c|c|}
\hline Municípios ${ }^{\star}$ & População & $\begin{array}{r}\text { n de equipes por } \\
\text { município }{ }^{\star \star}\end{array}$ & $\begin{array}{r}\text { n de equipes que aderiram } \\
\text { ao PMAQ-AB }{ }^{\star \star \star}\end{array}$ & $\begin{array}{r}\text { n de usuários respondentes } \\
\text { no PMAQ-AB }{ }^{\star \star \star}\end{array}$ \\
\hline Alenquer & 54.353 & 2 & 2 & 8 \\
\hline Almeirim & 33.466 & 6 & 3 & 13 \\
\hline Belterra & 16.924 & 5 & 5 & 20 \\
\hline Curuá & 13.333 & 4 & 1 & 4 \\
\hline Juruti & 52.755 & 7 & 6 & 23 \\
\hline Monte Alegre & 56.231 & 8 & 3 & 12 \\
\hline Óbidos & 50.317 & 5 & 2 & 8 \\
\hline Placas & 27.700 & 2 & 1 & 4 \\
\hline Prainha & 29.270 & 6 & 5 & 20 \\
\hline Santarém & 290.521 & 32 & 26 & 104 \\
\hline Terra Santa & 17.783 & 4 & 4 & 16 \\
\hline Total Geral & 642.653 & 81 & 58 & 232 \\
\hline \multicolumn{5}{|c|}{$\begin{array}{l}\text { *A região de saúde do Baixo Amazonas é composta por } 14 \text { municípios, no entanto, os municípios de Faro, Mojuí dos Campos e Oriximiná } \\
\text { não aderiram ao PMAQ-AB. }\end{array}$} \\
\hline \multicolumn{5}{|c|}{ **Sala de Apoio à Gestão Estratégica (Sage). Dados de março referente ao ano de 201710.} \\
\hline \multicolumn{5}{|c|}{ ***Banco do PMAQ-AB, ciclo 2, 2014 11. } \\
\hline
\end{tabular}

No eixo acesso (tabela 2), observaram-se os seguintes resultados, segundo respostas das equipes de saúde: $44,8 \%$ das equipes responderam que os usuários são agendados em qualquer dia da semana, em qualquer horário; $22,4 \%$, em dias específicos e em horários específicos; e 20,7\%, em qualquer dia da semana e em horários específicos. Geralmente, as equipes agendam esses usuários com hora marcada $(37,9 \%)$, ou marcam por bloco de horas $(36,2 \%)$, ou ainda há a necessidade de fila para pegar senha $(25,9 \%)$, sendo esse agendamento feito sempre de forma presencial. A utilização de fichas e filas aponta dificuldades na organização da agenda, distanciando-se da proposta de acolhimento com classificação de risco nas unidades de saúde da família (considerando risco aqui, também como social) e aponta para a prevalência de um modelo tradicional de pronto atendimento, centrado na consulta médica ${ }^{13,14}$.

Quanto ao tempo de espera do usuário desde sua chegada até o momento do primeiro acolhimento, $69,0 \%$ dos profissionais responderam que gira em torno de 10 a 30 minutos; e $29,3 \%$, de 31 a 60 minutos, evidenciando certa preocupação quanto à demora para o primeiro atendimento ao usuário. Isso porque, após o acolhimento, ainda é acrescido o tempo de espera do usuário até o momento da consulta, e essa avaliação se agrava ao refletir que acolhimento e consulta ainda não são garantia de resolutividade às necessidades de saúde do usuário. Além disso, a procura pelos serviços de saúde pode ser influenciada pelo tempo de espera, conforme demonstra Comes et al. ${ }^{15} \mathrm{em}$ seu estudo, em que tempo de espera reduzido é uma variável para maior satisfação dos usuários. 
Tabela 2. Resultados de acesso sob a perspectiva das equipes de saúde, PMAQ-AB região de saúde do Baixo Amazonas, Pará, 2014

\begin{tabular}{|c|c|c|c|}
\hline \multirow[t]{2}{*}{ Acesso/Equipes de Saúde } & & \multicolumn{2}{|c|}{ Baixo Amazonas } \\
\hline & & $\mathrm{n}$ & $\%$ \\
\hline \multirow{5}{*}{$\begin{array}{l}\text { EqAB: como são agendadas as consultas } \\
\text { na unidade? * }\end{array}$} & Em qualquer dia da semana, em qualquer horário & 26 & 44,8 \\
\hline & Em qualquer dia da semana, em horários específicos & 12 & 20,7 \\
\hline & Dias específicos fixos, em qualquer horário & 4 & 6,9 \\
\hline & Dias específicos fixos, em horários específicos & 13 & 22,4 \\
\hline & Outros & 3 & 5,2 \\
\hline \multirow[t]{3}{*}{ EqAB: como os usuários são agendados? } & Com hora marcada & 22 & 37,9 \\
\hline & Marcado por blocos de hora & 21 & 36,2 \\
\hline & Fila para pegar senha & 15 & 25,9 \\
\hline EqAB: o agendamento pode ser feito? & Presencial & 58 & 100,0 \\
\hline \multirow{3}{*}{$\begin{array}{l}\text { EqAB: tempo de espera do usuário desde } \\
\text { a chegada até o momento do primeiro } \\
\text { acolhimento }\end{array}$} & 10 a 30 minutos & 40 & 69,0 \\
\hline & 31 a 60 minutos & 17 & 29,3 \\
\hline & Mais de 60 minutos & 1 & 1,7 \\
\hline \multirow{3}{*}{$\begin{array}{l}\text { EqAB: tempo que o usuário espera para } \\
\text { a consulta }\end{array}$} & 10 a 30 minutos & 29 & 50,0 \\
\hline & 31 a 60 minutos & 17 & 29,3 \\
\hline & Mais de 60 minutos & 12 & 20,7 \\
\hline \multicolumn{2}{|c|}{$\begin{array}{l}\text { EqAB: o usuário consegue sair da unidade com a consulta marcada nas situações em que não seja o } \\
\text { caso atender no mesmo dia? }{ }^{1}\end{array}$} & 56 & 96,6 \\
\hline \multicolumn{4}{|c|}{ 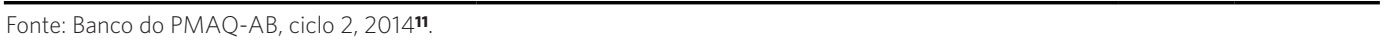 } \\
\hline \multicolumn{4}{|c|}{ *n de EqAB: Baixo Amazonas = 58.} \\
\hline \multicolumn{4}{|c|}{${ }^{1} \mathrm{O}$ percentual corresponde ao número de equipes que responderam sim. } \\
\hline \multicolumn{4}{|c|}{ EqAB= Equipe da Atenção Básica, PMAQ-AB= Programa Nacional de Melhoria do Acesso e da Qualidade da Atenção Básica. } \\
\hline
\end{tabular}

No eixo acolhimento (tabela 3), foram apresentados os resultados a partir dos aspectos: realização do acolhimento e frequência. Os resultados mostram que 98,3\% das equipes de saúde participantes do PMAQ-AB responderam que realizam o acolhimento à demanda espontânea, indicando a inserção dessa prática continuamente nos processos de trabalho, cabendo, entretanto, avaliar não apenas a existência, mas a qualidade do acolhimento. Nos ‘Cadernos de Atenção Básica' do Ministério da Saúde, é expresso que o acolher com qualidade não é só distribuir senhas em número limitado e fazer a triagem ao médico, é muito mais amplo, envolvendo a escuta de forma ampliada, o reconhecimento de riscos/ vulnerabilidades e a oferta de soluções para necessidades de saúde do usuário ${ }^{16}$.
A pesquisa de Clementino et al. ${ }^{17}$ traz a variação de percepções sobre o conceito e o modo de fazer acolhimento: para a maioria dos profissionais, o acolhimento é como se fosse apenas mais uma prática desenvolvida entre tantas outras atividades oferecidas pela $\mathrm{AB}$, enquanto para poucos seria um novo posicionar do profissional diante das necessidades do usuário.

Quanto ao local de realização do acolhimento: $59,6 \%$ dos profissionais colocaram a sala de recepção como principal espaço para o atendimento, seguido de consultório (15,8\%) e de nenhum outro local específico (10,5\%). Os dados mostram que, geralmente, os usuários são direcionados para a sala de recepção, contudo, sabe-se que o acolhimento deve acontecer nos diferentes ambientes da UBS. Braz $^{18}$ argumenta que o acolhimento não se 
restringe a um local ou sala específica, mas parte de uma postura humana e enfatiza que, em todo lugar onde ocorra um encontro entre qualquer profissional e usuário, deve ocorrer a prática de acolhimento, na qual promova a constituição de vínculos e responsabilização profissional.

Tabela 3. Resultados de acolhimento sob a perspectiva das equipes de saúde, PMAQ-AB região de saúde do Baixo Amazonas, Pará, 2014

\begin{tabular}{|c|c|c|c|}
\hline \multicolumn{2}{|l|}{ Acolhimento/Equipes de Saúde } & \multicolumn{2}{|c|}{ Baixo Amazonas } \\
\hline & & \multirow{3}{*}{$\begin{array}{c}\mathbf{n} \\
57\end{array}$} & \multirow{2}{*}{$\begin{array}{r}\% \\
98,3\end{array}$} \\
\hline \multicolumn{2}{|l|}{ EqAB que realiza o acolhimento à demanda espontânea } & & \\
\hline \multirow[t]{5}{*}{ EqAB: local de realização do acolhimento* } & Sala de espera/recepção & & 59,6 \\
\hline & Consultório & 9 & 15,8 \\
\hline & Não há local específico & 6 & 10,5 \\
\hline & Outro local & 5 & 8,8 \\
\hline & Sala específica & 3 & 5,3 \\
\hline \multirow[t]{3}{*}{ EqAB: em quais turnos é realizado o acolhimento ${ }^{\star 1}$} & Manhã & 54 & 95,0 \\
\hline & Tarde & 52 & 91,0 \\
\hline & Noite & 2 & 4,0 \\
\hline \multirow[t]{3}{*}{ EqAB: em quantos dias por semana acontece?* } & Um a dois dias por semana & 5 & 8,8 \\
\hline & Três a quatro dias por semana & 4 & 7,0 \\
\hline & Cinco ou mais dias por semana & 48 & 84,2 \\
\hline
\end{tabular}

Os turnos mais frequentes para o acolhimento, segundo os profissionais, são manhã com $95,0 \%$ e tarde com $91,0 \%$, sendo que, no turno noturno, $\mathrm{o}$ acolhimento acontece apenas em duas UBS do total geral (4,0\%). A maioria das UBS da região de saúde do Baixo Amazonas não realiza atendimento nos três turnos, o que poderia ser apontado como um limitador de acesso caso se considere a dificuldade dos usuários em frequentar a UBS em horário comercial. Contudo, apesar das unidades não atenderem os três turnos ou fins de semana, os usuários entrevistados avaliam positivamente o horário de atendimento quanto às suas necessidades de saúde. Em relação aos dias de atendimento, $84,2 \%$ dos profissionais informaram que o acolhimento acontece em cinco dias ou mais por semana, o que indica que essas UBS podem ser consideradas como as mais acessíveis (tabela 3). Na visão de $84,9 \%$ dos usuários entrevistados, o horário de funcionamento atende as suas necessidades de saúde (tabela 4).

Foram entrevistados pelo PMAQ-AB da região de saúde do Baixo Amazonas um total de 232 usuários. Para caracterizar o acesso, destacaram-se as variáveis: tempo (casa/ unidade, atendimento/consulta), funcionamento da unidade e marcação de consulta. Relacionado com o acesso (tabela 4), 90,9\% dos usuários responderam que levam de 10 a 30 minutos de casa até a sua unidade, e, na avaliação dos usuários, chegar até essa unidade está entre fácil (53,4\%), muito fácil (28,9\%) e 
razoável (12,5\%). A espera dos usuários para ser atendido por um profissional de saúde da $\mathrm{AB}$ varia de 10 a 30 minutos (37,8\%), de 31 a 60 minutos (23,9\%) a mais de 60 minutos (38,3\%). Entende-se que os referidos tempos de espera podem ser reduzidos, principalmente por esse ser um indicador de satisfação do usuário ${ }^{15} \mathrm{e}$ de qualidade do serviço.

No que se refere à marcação de consultas, 97,0\% dos usuários afirmam que as unidades de saúde a realizam. Contudo, a forma de marcação é variável: $62,2 \%$ dos usuários responderam que, para marcar consulta, é necessário ir à unidade e marcar o atendimento; $31,6 \%$ vai à unidade, mas tem que pegar ficha sem ficar na fila; $26,7 \%$ vai à unidade e fica na fila para pegar ficha; e em $17,3 \%$ o agente comunitário de saúde marca a consulta. $\mathrm{O}$ percentual de usuários que ainda precisam ficar nas filas e pegar fichas denota falhas na organização da agenda da equipe de saúde, o que pode ser observado no artigo de Souza et al. ${ }^{\mathbf{1 4}}$, que demonstra a existência de filas como dificultador de primeiro acesso, sejam essas filas físicas ou virtuais (em processos de UBS que passam pela informatização).

De acordo com os usuários entrevistados, a consulta pode ser realizada: em qualquer dia da semana, em qualquer horário (46,0\%), em dias específicos, em horários específicos $(30,8 \%)$ e em qualquer dia da semana, em horários específicos (17,9\%). Tais respostas podem ser equiparadas com a dos profissionais, sendo perceptível que uma grande parte dos usuários ainda precisa cumprir dia e horário específicos para a marcação de consultas (tabela 4). Esses dados, combinados com a necessidade de pegar ficha e fazer fila nas UBS, reafirmam uma necessidade de melhoria no atendimento à demanda espontânea.

Tabela 4. Resultados de acesso sob a perspectiva dos usuários, PMAQ-AB região de saúde do Baixo Amazonas, Pará, 2014

\begin{tabular}{|c|c|c|c|}
\hline \multirow[t]{2}{*}{ Acesso/Usuários } & & \multicolumn{2}{|c|}{ Baixo Amazonas } \\
\hline & & $\mathbf{n}$ & $\%$ \\
\hline \multirow{3}{*}{$\begin{array}{l}\text { Usuários: quanto tempo o senhor leva da sua casa } \\
\text { até a unidade? }\end{array}$} & 10 a 30 minutos & 211 & 90,9 \\
\hline & 31 a 60 minutos & 18 & 7,8 \\
\hline & Mais de 60 minutos & 3 & 1,3 \\
\hline \multirow{3}{*}{$\begin{array}{l}\text { O(a) senhor(a) sabe quanto tempo espera para ser } \\
\text { atendido por um profissional de saúde na maioria } \\
\text { das vezes?! }\end{array}$} & 10 a 30 minutos & 76 & 37,8 \\
\hline & 31 a 60 minutos & 48 & 23,9 \\
\hline & Mais de 60 minutos & 77 & 38,3 \\
\hline \multirow[t]{5}{*}{ Chegar até essa unidade é: } & Muito fácil & 67 & 28,9 \\
\hline & Fácil & 124 & 53,4 \\
\hline & Razoável & 29 & 12,5 \\
\hline & Difícil & 11 & 4,7 \\
\hline & Muito difícil & 1 & 0,4 \\
\hline \multirow[t]{3}{*}{ Em que horário a unidade funciona? ${ }^{\star}$} & Manhã & 230 & 99,1 \\
\hline & Tarde & 231 & 99,6 \\
\hline & Noite & 9 & 3,9 \\
\hline \multicolumn{2}{|c|}{ O horário de funcionamento atende às suas necessidades?* } & 197 & 84,9 \\
\hline \multicolumn{2}{|l|}{ Essa unidade faz marcação de consulta? ${ }^{\star \star}$} & 225 & 97,0 \\
\hline
\end{tabular}




\begin{tabular}{|c|c|c|c|}
\hline \multirow[t]{5}{*}{ Como é marcada a consulta?**2 } & Marca pela internet & 1 & 0,4 \\
\hline & Vai à unidade e marca atendimento & 140 & 62,2 \\
\hline & $\begin{array}{l}\text { Vai à unidade, mas tem que pegar ficha sem } \\
\text { ficar na fila }\end{array}$ & 71 & 31,6 \\
\hline & Vai à unidade e fica na fila para pegar ficha & 60 & 26,7 \\
\hline & $\begin{array}{l}\text { O agente comunitário de saúde marca a } \\
\text { consulta }\end{array}$ & 39 & 17,3 \\
\hline \multirow[t]{4}{*}{ A consulta normalmente pode ser feita: ${ }^{\star \star}$} & $\begin{array}{l}\text { Em qualquer dia da semana, em qualquer } \\
\text { horário }\end{array}$ & 103 & 46,0 \\
\hline & $\begin{array}{l}\text { Em qualquer dia da semana, em horários } \\
\text { específicos }\end{array}$ & 40 & 17,9 \\
\hline & Dias específicos, em qualquer horário & 14 & 6,3 \\
\hline & Dias específicos, em horários específicos & 69 & 30,8 \\
\hline
\end{tabular}

Fonte: Banco do PMAQ-AB, ciclo 2, 201411.

*n usuários PMAQ-AB: Baixo Amazonas = 232.

**n de usuários que responderam que a unidade faz marcação de consultas: Baixo Amazonas = 225

1 O n de usuários = 201, os 31 respondentes que faltam não souberam responder

2 Essa questão admite múltiplas respostas.

PMAQ-AB= Programa Nacional de Melhoria do Acesso e da Qualidade da Atenção Básica.

No acolhimento, na perspectiva dos usuários (tabela 5), foram observados os seguintes elementos: atendimento à demanda espontânea e resolução de problemas pelas equipes de saúde. Dos 232 usuários entrevistados, apenas $55,6 \%$ precisaram ir à unidade sem hora marcada; e para $87,6 \%$ dos usuários, foi realizado algo para resolver o seu problema na UBS. Percebe-se que cerca de $80 \%$ dos usuários afirmam terem sido bem recebidos na UBS. Contudo vê-se a necessidade de ampliar o conceito de 'bem recebido', já que a pergunta traz um nível de satisfação pouco qualitativo quanto à integralidade nesse atendimento. Em outras palavras, percebeu-se nas entrevistas dos usuários que, apesar de tecerem críticas ao atendimento, a resposta sobre 'bem recebidos' e a nota do atendimento na UBS, em geral, mostraram-se elevadas.

Quanto à equipe buscar resolver seus problemas na própria UBS, $50,2 \%$ dos usuários responderam positivamente; e 45,5\%, na maioria das vezes, apontando para uma tendência à resolutividade pelas equipes de AB. Nota-se também que, apesar de os usuários estarem dispostos a falar, mais de $60 \%$ responderam que os profissionais pouco perguntam sobre as questões da sua vida, fato que distorce o conceito do acolhimento como reorganização do processo de trabalho. Outra questão que pode ser relacionada a como está sendo 'implantado' o acolhimento é que mais de $50 \%$ dos usuários responderam que os profissionais não perguntam sobre questões da sua vida, mesmo se sentindo à vontade para falar com a equipe sobre as suas preocupações. Logo, é possível visualizar que, mesmo que os usuários estejam dispostos a falar, os profissionais pouco abrem espaços para que seja conhecido o contexto social do usuário. 
Tabela 5. Resultados de acolhimento sob a perspectiva dos usuários, PMAQ-AB região de saúde do Baixo Amazonas, Pará, 2014

\begin{tabular}{|c|c|c|c|}
\hline \multirow[t]{2}{*}{ Acolhimento/Usuários } & & \multicolumn{2}{|c|}{ Baixo Amazonas } \\
\hline & & $\mathrm{n}$ & $\%$ \\
\hline \multicolumn{2}{|l|}{ O senhor já precisou ir à unidade sem hora marcada?* } & 129 & 55,6 \\
\hline \multirow[t]{5}{*}{ Como o senhor foi recebido $?^{\star \star}$} & Muito bem & 23 & 17,8 \\
\hline & Bem & 80 & 62,0 \\
\hline & Regular & 20 & 15,5 \\
\hline & Ruim & 2 & 1,6 \\
\hline & Muito ruim & 4 & 3,1 \\
\hline \multicolumn{2}{|l|}{ Foi realizado algo para resolver seu problema?** } & 113 & 87,6 \\
\hline \multirow{4}{*}{$\begin{array}{l}\text { A equipe busca resolver suas necessidades/problemas na própria } \\
\text { unidade de saúde? }\end{array}$} & Sim & 116 & 50,2 \\
\hline & Na maioria das vezes & 105 & 45,5 \\
\hline & Quase nunca & 8 & 3,5 \\
\hline & Nunca & 2 & 0,9 \\
\hline \multirow{4}{*}{$\begin{array}{l}\text { Os profissionais de saúde perguntam sobre outras questões da sua } \\
\text { vida? }\end{array}$} & Sim & 106 & 46,1 \\
\hline & Na maioria das vezes & 48 & 20,9 \\
\hline & Quase nunca & 16 & 7,0 \\
\hline & Nunca & 60 & 26,1 \\
\hline \multirow{4}{*}{$\begin{array}{l}\text { O(a) senhor (a) se sente à vontade para falar com a equipe sobre } \\
\text { suas preocupações? }\end{array}$} & Sim & 160 & 69,3 \\
\hline & Na maioria das vezes & 34 & 14,7 \\
\hline & Quase nunca & 10 & 4,3 \\
\hline & Nunca & 27 & 11,7 \\
\hline
\end{tabular}

Fonte: Banco do PMAQ-AB, ciclo 2, 2014"1.

${ }^{*}$ n usuários PMAQ-AB: Baixo Amazonas = 232.

${ }^{*} n$ de usuários que precisaram ir à unidade sem hora marcada: Baixo Amazonas = 129.

PMAQ-AB= Programa Nacional de Melhoria do Acesso e da Qualidade da Atenção Básica.

Clementino et al. ${ }^{\mathbf{1 7}}$, em seu estudo sobre o acolhimento no Brasil, colocaram dois aspectos que podem explicar a diferença entre as respostas de profissionais e de usuários: 1) divergência de percepção sobre que é ser acolhido e ser ouvido e 2) produção de pouco impacto no modo do usuário visualizar a atenção em saúde, ora quando não concretizado de fato o acolhimento pelas equipes de saúde da família.

Se tal postura é problemática na organização da $\mathrm{AB}$ para atendimento integral da população, o quadro se agrava quando se refere a populações específicas, como, por exemplo, a População do Campo, da Floresta e das Águas, usuários de saúde mental ou população de Lésbicas, Gays, Bissexuais, Travestis, Transexuais (LGBTT), muitas vezes invisibilizadas no SUS. Figueira et al. ${ }^{19}$ demonstram que existem dificuldades, como a distância entre a comunidade e os serviços; a rotatividade dos profissionais nas UBS, devido à infraestrutura da comunidade e à subestimação dos profissionais quanto à eficácia dos remédios caseiros, entre outras, para que estas populações tenham acesso aos serviços de saúde, e quando estes estão disponíveis, há problemas na sua qualidade. Tal fato sugere o fortalecimento 
da força de trabalho em saúde e da formação dos profissionais, sendo esses alocados estrategicamente nas regiões para maiores possibilidades de garantia de atendimento às populações vulneráveis.

O envolvimento do usuário no próprio cuidado pode fortalecer a capacidade de relação e vínculo com a equipe, sendo essencial que esse exponha a sua percepção acerca da atenção à saúde prestada. Alguns elementos destacam a visão dos usuários sobre o acolhimento desenvolvido nas UBS: a resolutividade nos serviços ofertados e a escuta ampliada. Cerca de 50\% dos usuários entrevistados afirmaram ter respostas para as suas necessidades/problemas na própria UBS. Nessa afirmação, apesar de ser um número expressivo na resolutividade dos problemas dos usuários, não se atinge o percentual indicado pelos profissionais de $98,3 \%$ de realização do acolhimento nas UBS, já que o acolhimento é conferido como proposta para reorganização dos serviços, promovendo o acesso universal, resolutividade e humanização no atendimento.

Assim, observa-se uma estreita relação entre acesso e acolhimento, porque embora se percebam avanços e facilidades na disponibilidade de serviços próximos à casa dos usuários, identificam-se fatores que determinam qual vai ser a escolha, tanto do serviço de saúde como do profissional, aspectos ligados à forma como o usuário é recebido e à resolutividade dos serviços. Dessa forma, a AB só será efetiva quando a produção de saúde for compartilhada entre a equipe de saúde e os usuários e, em decorrência disso, ocorra a mudança na visão de como produzir saúde e do papel da $\mathrm{AB}$ no cuidado ao usuário.

\section{Considerações finais}

Em geral, o estudo realizado apresentou resultados positivos. Alguns dos avanços notáveis estão relacionados com as formas de agendamento; fácil acesso do usuário à UBS (distância casa e unidade); horário de funcionamento atendendo às necessidades do usuário; mais 90\% de acolhimento implementado no processo de trabalho das equipes de saúde e a realização do atendimento nos cinco dias ou mais da semana.

Entretanto, estes resultados ainda mostram limitações para concretização do acesso e realização do acolhimento na região de saúde do Baixo Amazonas, entre elas: dificuldades na organização da agenda, destacando que ainda há a necessidade de fichas e fila para que o usuário chegue ao atendimento; tempos de espera que, quando altos, dificultam a integralidade do cuidado e impactam na satisfação do usuário; turnos de atendimento, considerando a dificuldade dos usuários em frequentar a UBS em horário comercial; concentração do acolhimento em apenas um ambiente da unidade; qualidade do acolhimento, visto que este é mais do que uma triagem ao médico; e obstáculos no processo de escuta e na resolutividade das necessidades de saúde dos usuários.

Uma observação a ser feita é que, em geral, os profissionais apresentaram uma avaliação mais positiva do que os usuários quanto às dimensões pesquisadas. Essa questão pode se referir ao modo como as orientações recebidas estão atendendo às expectativas dos usuários, sem produzir maior impacto na saúde. Já que a visão do usuário nesta pesquisa apresenta-se em partes divergentes a do profissional, caberia aqui avaliar os recursos que faltam para que o usuário visualize a $\mathrm{AB}$ integralmente como primeiro acesso.

É importante ressaltar que o PMAQ-AB pode ser considerado o maior programa nacional de avaliação existente no Brasil, permitindo um amplo diagnóstico e uma série de melhorias no processo de trabalho da $\mathrm{AB}$, seja no âmbito dos serviços, seja na produção científica da área. Entretanto, dos resultados encontrados, problematiza-se a questão do acesso, visto que os usuários respondentes/ participantes do programa previamente já acessaram a UBS, sendo este um critério do PMAQ-AB: usuários que estavam na UBS na hora da entrevista. Ou seja, os resultados 
encontrados de acesso expõem a realidade de um público que já acessou a UBS.

Diante dessas constatações, indaga-se: considerando as equipes que aderiram ao PMAQ-AB, seria o incentivo financeiro um potencializador de mudanças de práticas de saúde? Considerando as equipes que não aderiram ao PMAQ-AB, quais as dificuldades que estas equipes sem incentivo nenhum estão tendo?

Por mais que o acesso e o acolhimento estejam afirmados na $A B$, ainda é preciso ações integradas na produção de saúde, que gerem o envolvimento e a satisfação do usuário. Acredita-se que o fortalecimento da regionalização no Baixo Amazonas pode criar condições de suporte estrutural e organizacional para que os municípios possam atuar de forma mais integrada e resolutiva aos problemas dos usuários.

\section{Colaboradores}

Silva LAN (0000-0003-4287-5844)* e Lima JG (0000-0002-5576-0002)* contribuíram para a concepção, planejamento, análise, interpretação dos dados e aprovação da versão final do manuscrito. Harayama RM (0000-0002-72313589)* e Fernandes FP (0000-0002-4617-1919)* contribuíram para a interpretação dos dados, revisão crítica do conteúdo e aprovação da versão final do manuscrito.

\section{Referências}

1. Starfield B. Atenção primária: equilíbrio entre necessidades de saúde, serviços e tecnologia. Brasília, DF: Unesco/Ministério da Saúde; 2002.

2. Franco TB, Bueno WS, Merhy EE. O acolhimento e os processos de trabalho em saúde: Betim, Minas Gerais, Brasil. Cad. Saúde Pública. 1990; 15(2):345-353.

3. Malta DC, Ferreira LM, Reis AT, et al. Mudando o processo de trabalho na rede pública: alguns resultados da experiência em Belo Horizonte. Saúde debate [internet]. 2000 [acesso em 2018 jan 21]; 24(56):2134. Disponível em: http://www.scielo.br/scielo. php?script=sci_nlinks\&ref=000146\&pid=S0102$-311 X 200800130001500011 \& \operatorname{lng}=\mathrm{en}$.
4. Santos L, Campos GWS. SUS Brasil: A região de saúde como caminho. Saúde Soc [internet]. 2015 [acesso em 2018 jan 10]; 24(2):438-446. Disponível em: http://www.scielo.br/scielo.php?pid=S0104$-12902015000200438 \&$ script=sci_abstract\&tlng=pt.

5. Fernandes FMB. Regionalização no SUS: uma revisão crítica. Ciênc. Saúde Colet. 2017; 22(4):1311-1320.

6. Garnelo L, Sousa ABL, Silva CO. Regionalização em saúde no Amazonas: avanços e desafios. Ciênc. Saúde Colet. 2017; 22(4):1225-1234.

7. Viana ALD, Bousquat A, Pereira APCM, et al. Tipologia das regiões de saúde: condicionantes estruturais para a regionalização no Brasil. Saúde Soc [internet].
* Orcid (Open Researcher and Contributor ID). 
2015 [acesso em 2018 jan 10]; 24(2):413-422. Disponível em: http://www.scielo.br/scielo.php?script=sci arttext\&pid=S0104-12902015000200413.

8. Santos L. Região de saúde e suas redes de atenção: modelo organizativo-sistêmico do SUS. Ciênc. Saúde Colet. 2017; 22(4):1281-1289.

9. Mendes A, Louvison MCP, Ianni AMZ, et al. O processo de construção da gestão regional da saúde no estado de São Paulo: subsídios para a análise. Saúde Soc [internet]. 2015 [acesso em 2018 jan 15]; 24(2):423437. Disponível em: http://www.scielo.br/scielo. php?pid=S0104-12902015000200423\&script=sci abstract\&tlng=pt.

10. Brasil. Ministério da Saúde. SAGE - Sala de Apoio à Gestão Estratégica. Dados de março referente ao ano de 2017 [internet]. 2017 [acesso em 2018 jan 10]. Disponível em: http://sage.saude.gov.br.

11. Brasil. Ministério da Saúde, Secretaria de Atenção à Saúde, Departamento de Atenção Básica. Instrumento de avaliação externa paras as equipes de Atenção Básica - PMAQ-AB [internet]. 2013 [acesso em 2017 nov 22]. Disponível em: http://dab.saude.gov.br/portaldab/ape_pmaq.php?conteudo=2_ciclo.

12. Lima JG, Giovanella L, Fausto MCR, et al. Atributos essenciais da Atenção Primária à Saúde: resultados nacionais do PMAQ-AB. Saúde debate [internet] 2018 [acesso em 2018 fev 15]. 42(esp1):52-66. Disponível em: http://www.scielo.br/scielo.php?script=sci abstract\&pid=S0103-11042018000500052\&lng=en\& nrm=iso\&tlng=pt.

13. Fausto MCR, Giovanella L, Mendonça MHM, et al. A posição da Estratégia Saúde da Família na rede de atenção à saúde na perspectiva das equipes e usuários participantes do PMAQ-AB. Saúde debate [internet]. 2014 [acesso em 2018 jan 12]; 38(esp):13-33. Disponí- vel em: http://www.scielo.br/scielo.php?pid=S0103$-11042014000600013 \&$ script=sci_abstract $\&$ tlng=pt.

14. Souza ECF, Vilar RLA, Rocha NSPD, et al. Acesso e acolhimento na atenção básica: uma análise da percepção dos usuários e profissionais de saúde. Cad. Saúde Pública. 2008; 24(sup1):S100-S110.

15. Comes Y, Trindade JS, Shimizu HE, et al. Avaliação da satisfação dos usuários e da responsividade dos serviços em municípios inscritos no Programa Mais Médicos. Ciênc. Saúde Colet. 2016; 21(9):2749-2759.

16. Brasil. Ministério da Saúde. Cadernos de Atenção Básica: Acolhimento a demanda espontânea n. 28. Brasília, DF: Ministério da Saúde; 2011. Volume 1.

17. Clementino FS, Gomes LB, Vianna RPT, et al. Acolhimento na Atenção Básica: Analise a partir da avaliação externa do Programa de Melhoria do Acesso e da Qualidade da Atenção Básica (PMAQ-AB). Rev. Saúde Ciênc. 2015; 4(1):62-80.

18. Braz FAF. A importância do acolhimento aos usuários na Atenção Básica: uma assistência humanizada. [monografia]. Belo Horizonte: Universidade Federal de Minas Gerais; 2013. 46 p.

19. Figueira MCS, Silva WP, Silva EM. Acesso aos serviços da Atenção Primária em Saúde: revisão integrativa da literatura. Rev Bras Enferm [internet]. 2018 [acesso em 2018 fev 20]; 71(3):1246-1257. Disponível em: http://www.scielo.br/scielo.php?script=sci arttext\&pid=S0034-71672018000301178\&lng=pt\&n $\mathrm{rm}=\mathrm{iso \& t} \operatorname{lng}=\mathrm{pt}$.

Recebido em 09/04/2019

Aprovado em 24/08/2019

Conflito de interesses: inexistente

Suporte financeiro: Edital no 08/2017 - Programa de Fomento

a Trabalhos de Conclusão de Curso da Universidade Federal do Oeste do Pará (Ufopa) 